ISSN 1991- 8690

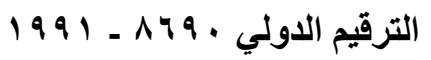

Website: http://jsci.utq.edu.iq

Email: utjsci@utq.edu.iq

\title{
The variational iterated method for solving integral equations
}

\author{
Abeer A. A. Aladhab \\ College of Art - Thi-Qar University
}

\section{$\underline{\text { Abstract }}$}

The main objective of this paper is to study the exact solution and approximate solution type of integral equations, by using the variational iteration method, as well as, giving some illustrative examples of linear and nonlinear equations. We tabulate the exact and approximate results.

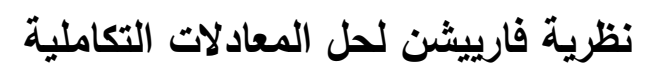

$$
\text { كلية الآداب - جبد الكاظم عبد الرزاق ذبير }
$$

\section{الخلاصة:}

تتاولنا في هدا البحث الحلول الحقيقية والتقريبية للمعادلات التكاملية باستخدام طريقة( فارييشن اترايتد )،وقدمنا بعض الامنلة الخطية والغير خطية . وقدمنا النتائج في جداول.

\section{Introduction, $,[1],[3]$}

In some cases, the analytical solution may be difficult to evaluate, therefore numerical and approximate methods are needed. The numerical method that will be considered in this work is the variational iteration method (which is abbreviated by VIM) for finding the solution of linear and nonlinear problems. This method is a modification of the general Lagrange multiplier method into an iteration method, which is called the correction functional. Heuristic interpretation of those concepts leads to new comers in the field to start working immediately without the long search and preparation of advanced calculus and calculus of variations, at the same time those concepts already familiar with variational iteration method which will find the most recent new result. In 1998, he solved the classical blasius eqation using VIM.in 1999, he used VIM to give approximate solution for some well-known non -linear problems.in 2000, he used VIM to solve autonomous ordinary differential systems. In 2006 the VIM has recently been applied for solving nonlinear coagulation problem with mass loss by abulwafa and momani. In this paper, we apply the variational iteration method to solve the integral equation of the form

$$
\mathrm{U}(\mathrm{x})=\mathrm{f}(\mathrm{x})+\int \mathrm{k} 1(\mathrm{x}, \mathrm{s}) \mathrm{u}(\mathrm{s}) \mathrm{ds}+\int \mathrm{k} 2(\mathrm{x}, \mathrm{s}) \mathrm{u}(\mathrm{s}) \mathrm{ds}
$$

Where $\mathrm{f}(\mathrm{x}), \mathrm{k} 1, \mathrm{k} 2$ be a continous function.

\section{Variational Iteration Method, [2],[4]}

Variational iteration method which was proposed by Ji-Huan in 1998 has been recently and intensively studied by several scientists and engineers, favorably applied to various kinds of linear and nonlinear problems. To illustrate the basic idea of the VIM, we consider the following general non-linear equation given in operator form: 


$$
\mathrm{L}(\mathrm{u}(\mathrm{x}))+\mathrm{N}(\mathrm{u}(\mathrm{x}))=\mathrm{g}(\mathrm{x}), \mathrm{x} \in[\mathrm{a}, \mathrm{b}]
$$

where $\mathrm{L}$ is a linear operator, $\mathrm{N}$ is a nonlinear operator and $\mathrm{g}(\mathrm{x})$ is any given function which is called the nonhomogeneous term.

Now, rewrite eq.(2) in a manner similar to eq.(2) as follows:

$$
\mathrm{L}(\mathrm{u}(\mathrm{x}))+\mathrm{N}(\mathrm{u}(\mathrm{x}))-\mathrm{g}(\mathrm{x})=0
$$

and let un be the nth approximate solution of eq. (3), then it follows that:

$$
\mathrm{L}(\mathrm{un}(\mathrm{x}))+\mathrm{N}(\mathrm{un}(\mathrm{x}))-\mathrm{g}(\mathrm{x}) \neq 0
$$

and then the correction functional for (2) is given by:

$$
\mathrm{u}_{\mathrm{n}+1}(\mathrm{x})=\mathrm{u}_{\mathrm{n}}(\mathrm{x})+\int_{\mathrm{x}_{0}}^{\mathrm{x}} \lambda(\mathrm{s})\left\{\mathrm{L}\left(\mathrm{u}_{\mathrm{n}}(\mathrm{s})+\mathrm{N}\left(\tilde{\mathrm{u}}_{\mathrm{n}}(\mathrm{s})\right)-\mathrm{g}(\mathrm{s})\right\} \mathrm{ds}\right.
$$

where $\lambda$ is the general Lagrange multiplier which can be identified optimally via the variational theory, the subscript $n$ denotes the nth approximation of the solution $u$ and $\tilde{u}_{n}$ is considered as a restricted variation, i.e., $\delta \tilde{u}_{n}=0$.

To solve eq. (5) by the VIM, we first determine the Lagrange multiplier $\lambda$ that will be identified optimally via integration by parts. Then the successive approximation $\mathrm{un}(\mathrm{x}), \mathrm{n}=0,1, \ldots$; of the solution $\mathrm{u}(\mathrm{x})$ will be readily obtained upon using the obtained Lagrange multiplier and by using any selective function $\mathrm{u} 0(\mathrm{x})$. The zeroth approximation $\mathrm{u} 0$ may be selected by any function that just satisfies at least the initial and boundary conditions with $\lambda$ determined, then several approximations un(x), $\mathrm{n}=0,1, \ldots$; follow immediately, and consequently the exact solution may be arrived since:

$$
u(x)=\lim _{n \rightarrow \infty} u_{n}(x)
$$

In other words, the correction functional for eq. (5) will give several approximations, and therefore the exact solution is obtained as the limit of the resulting successive approximations.

\section{Illustrative Examples}

In this section, some examples are given to illustrate the applicability and efficiency of the VIM for solving different types of problems.

\section{Example (1)}

Consider the linear integral equation,

$\mathrm{U}(\mathrm{x})=\mathrm{x} 2+4 \mathrm{x}+1 / 4+(\mathrm{x} 2+2 \mathrm{x}) \mathrm{ex}+\int \mathrm{k} 1(\mathrm{x}, \mathrm{s}) \mathrm{u}(\mathrm{s}) \mathrm{ds}+\int \mathrm{k} 2(\mathrm{x}, \mathrm{s}) \mathrm{u}(\mathrm{s}) \mathrm{ds} \ldots(7)$

With exact solution $u=x 2+2 x, u(0)=0$,

$\mathrm{K} 1=\mathrm{x}+\mathrm{s}, \mathrm{k} 2=\mathrm{xes}$.

\section{Solution:}

First, differentiate equation (7) with respect to $x$ yields:

$u^{\prime}(x)=2 x+4+(x 2+2 x) e x+e x(2 x+2)+\int(2 s+2 s 2) d s+\int e s(s 2+2 s) d s$

then, the following correction functional for eqation (8) may be obtained for all $n=0,1, \ldots$

$\mathrm{un}+1=\mathrm{un}(\mathrm{x})+\int \lambda(\mathrm{t})(\mathrm{L}(\mathrm{u} \mathrm{n}(\mathrm{t})-\mathrm{N}(\mathrm{un}(\mathrm{t})-\mathrm{g}(\mathrm{t})) \mathrm{dt}$

$u n+1=u n(x)+\lambda \lambda(t)\left\{u^{\prime}(x)-2 t-4-(t 2+2 t) e t-e t(2 t+2)-\int(2 s+2 s 2) d s-\int e s(s 2+2 s) d s \quad\right\} d t \ldots(10)$

where $\lambda$ is the general Lagrange multiplier . 
thus by taking the first variation with respect to the independent variable un and noticing that $\partial \mathrm{un}(0)=0$, we get $\partial u n+1(s)=\partial u n(s)+\partial \int \lambda(t)\left\{u^{\prime}(x)-2 t-4-(t 2+2 t) e t-e t(2 t+2)-\int(2 s+2 s 2) d s-\int e s(s 2+2 s) d s \quad\right\} d t$

where un is considered as a restricted variation, which means $\partial \mathrm{un}=0$ and consequently :

$\partial \mathrm{un}+1=\partial \mathrm{un}(\mathrm{x})+\partial \int \lambda(\mathrm{t})\left\{\mathrm{u}^{\prime} \mathrm{n}(\mathrm{t})\right\} \mathrm{dt}$

and by using the method of integration by parts, equation (11) will be reduced to

$\partial \mathrm{un}+1=\partial \mathrm{un}(\mathrm{x})+\lambda(\mathrm{t}) \partial \mathrm{un}(\mathrm{t}) \mid \mathrm{t}=\mathrm{x}-\int \lambda^{\prime}(\mathrm{t}) \partial \mathrm{un}(\mathrm{t}) \mathrm{dt}$

Hence:

$\partial \mathrm{un}+1(\mathrm{x})=1+\lambda(\mathrm{t}) \mid \mathrm{t}=\mathrm{x} \partial \mathrm{un}(\mathrm{x})-\int \lambda^{\prime} \partial \mathrm{un}(\mathrm{t}) \mathrm{dt}=0$

As a result, we have the following stationary conditions:

$\lambda^{\prime}(\mathrm{t})=0$

with natural boundary condition,

$1+\lambda(\mathrm{t}) \mid \mathrm{t}=\mathrm{x}=0$,

which is easily solved to give the lagrange multiplier $\lambda(t)=-1$. Now, substituting $\lambda(s)=-1$ back in to equation (10) give for all $\mathrm{n}=0,1, \ldots$

$\mathrm{Un}+1=\mathrm{un}(\mathrm{x})-\int\left\{\mathrm{u}^{\prime} \mathrm{n}(\mathrm{t})-2 \mathrm{t}-4-(\mathrm{t} 2+2 \mathrm{t}) \mathrm{et}-(2 \mathrm{t}+2) \mathrm{et}-\int(2 \mathrm{~s}+2 \mathrm{~s} 2) \mathrm{ds}-\int \mathrm{es}(\mathrm{s} 2+2 \mathrm{~s}) \mathrm{ds}\right\} \mathrm{dt}$

$\mathrm{U} 1=(-0.41) \mathrm{x}-\mathrm{x} 3 / 3$

$\mathrm{U} 2=\mathrm{u} 1(\mathrm{x})-\int\left\{\mathrm{u}^{\prime} 1(\mathrm{t})-2 \mathrm{t}-4-(\mathrm{t} 2+2 \mathrm{t}) \mathrm{et}-(2 \mathrm{t}+2) e t-\int(2 \mathrm{~s}+2 \mathrm{~s} 2) \mathrm{ds}-\int \mathrm{es}(\mathrm{s} 2+2 \mathrm{~s}) \mathrm{ds}\right\} \mathrm{dt}$

$\mathrm{U} 2=\mathrm{x} 2+4 \mathrm{x}+\mathrm{x}(\mathrm{x}-2) \mathrm{ex}$

$\mathrm{U} 3=\mathrm{u} 2(\mathrm{x})-\int\left\{\mathrm{u}^{\prime} 2(\mathrm{t})-2 \mathrm{t}-4-(\mathrm{t} 2+2 \mathrm{t}) \mathrm{et}-(2 \mathrm{t}+2) \mathrm{et}-\int(2 \mathrm{~s}+2 \mathrm{~s} 2) \mathrm{ds}-\int \mathrm{es}(\mathrm{s} 2+2 \mathrm{~s}) \mathrm{ds}\right\} \mathrm{dt}$ $\mathrm{U} 3=(3 \mathrm{x} 2-2 \mathrm{x}-2 \mathrm{x} 3-2 \mathrm{x} 4-\mathrm{x} 5) \mathrm{ex}-16 \mathrm{x}$

Table (1)

\begin{tabular}{|c|c|c|c|}
\hline $\mathrm{x}$ & $\left|\mathrm{u}(\mathrm{x})-\mathrm{u}_{1}(\mathrm{x})\right|$ & $|\mathrm{u}(\mathrm{x})-\mathrm{u}(\mathrm{x})|$ & $\left|u(\mathrm{x})-\mathrm{u}_{9}(\mathrm{x})\right|$ \\
\hline 0 & 0 & 0 & 0 \\
\hline 0.1 & 0.2513 & 0.1499 & 0.4014 \\
\hline 0.2 & 0.5222 & 0.761 & 0.2057 \\
\hline 0.3 & 0.822 & 0.871 & 0.9331 \\
\hline 0.4 & 1.1453 & 1.446 & 1.5195 \\
\hline 0.5 & 1.4966 & 1.764 & 1.6331 \\
\hline 0.6 & 1.878 & 1.662 & 1.3793 \\
\hline 0.7 & 2.291 & 2.369 & 2.4834 \\
\hline 0.8 & 2.738 & 2.664 & 2.1956 \\
\hline 0.9 & 3.222 & 3.866 & 3.123 \\
\hline 1 & 3.743 & 3.282 & 3.5872 \\
\hline
\end{tabular}

\section{Example(2)}

Consider the nonlinear integral equation,

$$
\mathrm{U}(\mathrm{x})=2 \mathrm{x} / 3-\mathrm{x} 3 / 6+\int \mathrm{k} 1(\mathrm{u}(\mathrm{t})) 2 \mathrm{dt}+\int \mathrm{k} 2(\mathrm{u}(\mathrm{t})) 2 \mathrm{dt}
$$


$\mathrm{u}(0)=0, \mathrm{x} \in[0,1]$

where $\mathrm{k} 1=\mathrm{xt}, \mathrm{k} 2=\mathrm{x}-\mathrm{t}, \mathrm{u}(\mathrm{x})=\mathrm{x}$ is the exact solution of equation (11)

\section{Solution:}

First, differentiate equation (11) with respect to $\mathrm{x}$

$u^{\prime}(x)=2 / 3-3 x 2 / 6+\int t(u(t)) 2 d t+\int(u(t)) 2 d t$

then by ( VIM ),

$\mathrm{un}+1(\mathrm{x})=\mathrm{un}(\mathrm{x})+\int \lambda(\mathrm{s})\left\{\mathrm{u}^{\prime} \mathrm{n}(\mathrm{s})-2 / 3-3 \mathrm{x} 2 / 6-\int \mathrm{t}(\mathrm{un}(\mathrm{t})) 2 \mathrm{dt}-\int(\mathrm{un}(\mathrm{t})) 2 \mathrm{dt}\right\} \mathrm{ds}$

where $\lambda$ is the general lagrange multiplier.

Thus, by taking the first variation with respect to the independent variable un and noting that $\partial$ un $(0)=0$, we get :

$\partial \mathrm{un}+1(\mathrm{t})=\partial \mathrm{un}(\mathrm{t})+\partial \int \lambda(\mathrm{s})\left\{\mathrm{un}^{\prime}(\mathrm{s})-2 / 3+3 \times 2 / 6-\int \mathrm{t}(\mathrm{un}(\mathrm{t})) 2 \mathrm{dt}-\int(\mathrm{un}(\mathrm{t})) 2 \mathrm{dt} \quad\right\} \mathrm{ds}$

where un is considered as a variation, which means $\partial \mathrm{un}=0$

$\partial \mathrm{un}+1=\partial \mathrm{un}(\mathrm{x})+\partial \int \lambda(\mathrm{s}) \mathrm{u}^{\prime} \mathrm{n}(\mathrm{s}) \mathrm{ds}$

and by using the method of integration by parts, equation (15) will be reduced to :

$\partial \mathrm{un}+1=\partial \mathrm{un}(\mathrm{x})+\lambda(\mathrm{s}) \partial \mathrm{un}(\mathrm{s}) \mid \mathrm{s}=\mathrm{x}-\int \lambda^{\prime}(\mathrm{s}) \partial \mathrm{un}(\mathrm{s}) \mathrm{ds}$

Hence:

$\partial \mathrm{un}+1=1+\lambda(\mathrm{s}) \mid \mathrm{s}=\mathrm{x} \partial(\mathrm{x})-\int \lambda^{\prime}(\mathrm{s}) \partial \mathrm{un}(\mathrm{s}) \mathrm{ds}=0$

As a result, we have

$\lambda^{\prime}(s)=0$

with natural boundary condition $\quad 1+\lambda(s) \mid s=x=0$

so $\lambda(s)=-1$

Now, substituting $\lambda(s)=-1$ back in to equation (13) gives for all $n=0,1, \ldots$

$\mathrm{un}+1(\mathrm{x})=\mathrm{un}(\mathrm{x})-\int\left\{\mathrm{u} \mathrm{n}^{\prime}(\mathrm{s})-2 / 3-3 \mathrm{x} 2 / 6-\int \mathrm{t}(\mathrm{u} \mathrm{n}(\mathrm{t})) 2 \mathrm{dt}-\int(\mathrm{u} \mathrm{n}(\mathrm{t})) 2 \mathrm{dt} \quad\right\} \mathrm{ds} \quad \ldots(16)$

let the initial approximate solution be

$\mathrm{u} 0=2 \mathrm{x} / 3-\mathrm{x} 3 / 6$

then

$$
\begin{aligned}
& \mathrm{u} 1(\mathrm{x})=\mathrm{u} 0(\mathrm{x})-\int\left\{\mathrm{u} 0^{\prime}(\mathrm{s})-2 / 3-3 \times 2 / 6-\int \mathrm{t}(\mathrm{u} 0(\mathrm{t})) 2 \mathrm{dt}-\int(\mathrm{u} 0(\mathrm{t})) 2 \mathrm{dt}\right\} \mathrm{ds} \\
& =0.748 \mathrm{x}+0.273 \mathrm{x} 4+0.039 \mathrm{x} 6 \\
& \begin{aligned}
\mathrm{U} 2(\mathrm{x}) & =\mathrm{u} 1(\mathrm{x})-\int\left\{\mathrm{u} 1^{\prime}(\mathrm{s})-2 / 3-3 \times 2 / 6-\int \mathrm{t}(\mathrm{u} 1(\mathrm{t})) 2 \mathrm{dt}-\int(\mathrm{u} 1(\mathrm{t})) 2 \mathrm{dt}\right\} \mathrm{ds} \\
= & 0.248 \times 3-0.0581 \mathrm{x}+0.39 \mathrm{x} 6
\end{aligned} \\
& \mathrm{U} 3(\mathrm{x})=\mathrm{u} 2(\mathrm{x})-\int\left\{\mathrm{u} 2^{\prime}(\mathrm{s})-2 / 3-3 \times 2 / 6-\int \mathrm{t}(\mathrm{u} 2(\mathrm{t})) 2 \mathrm{dt}-\int(\mathrm{u} 2(\mathrm{t})) 2 \mathrm{dt}\right\} \mathrm{ds} \\
& =0.6272 \mathrm{x}-0.062 \mathrm{x} 4+0.091 \times 6
\end{aligned}
$$

The absolute error between the exact and approximate solution of example (2) 
Table (2)

\begin{tabular}{|c|c|c|c|}
\hline $\mathrm{x}$ & $\left|\mathrm{u}(\mathrm{x})-\mathrm{u}_{1}(\mathrm{x})\right|$ & $\left|\mathrm{u}(\mathrm{x})-\mathrm{u}_{2}(\mathrm{x})\right|$ & $\left|\mathrm{u}(\mathrm{x})-\mathrm{u}_{3}(\mathrm{x})\right|$ \\
\hline 0 & 0 & 0 & 0 \\
\hline 0.1 & 0.0252 & 0.1055 & 0.0372 \\
\hline 0.2 & 0.0499 & 0.3184 & 0.0743 \\
\hline 0.3 & 0.0733 & 0.4829 & 0.111164 \\
\hline 0.4 & 0.094 & 0.4459 & 0.148014 \\
\hline 0.5 & 0.109 & 0.84306 & 0.18355 \\
\hline 0.6 & 0.117 & 0.9015 & 0.21941 \\
\hline 0.7 & 0.1093 & 0.3572 & 0.25622 \\
\hline 0.8 & 0.085 & 0.6027 & 0.29601 \\
\hline 0.9 & 0.032 & 0.8817 & 0.3425 \\
\hline 1 & 0.05 & 0.302 & 0.401 \\
\hline
\end{tabular}

\section{Reference :}

[1] Al-Amery H. A., "Variational Formulation for Solving Three-Dimensional Free and Moving Boundary Value Problems", M.Sc., Thesis, College of Education, Al-Mustansiriyah University, 2006.

[2] Batiha B., Noorani M. S. and Hashim I., "Numerical Solutions of The Nonlinear Integro-Differential Equations", Journal of Open Problems Compt. Math., Vol.1, No.1, pp. 34-41, 2008.

[3] He J-H., "Variational Iteration Method; A Kind of Non-Linear Analytical Technique; Some Examples", International Journal of Non-Linear Mechanics, 34, pp. 699-708, 1999.

[4] Xu L., "Variational Iteration Method for Solving Integral Equations", Journal of Computers and Mathematics with Applications, 54, pp.1071-1078, 2007. 